\title{
Localization of cortico-peripheral coherence with electroencephalography
}

\author{
Adrian G. Guggisberga,*, Sarang S. Dalal ${ }^{\mathrm{b}}$, Johanna M. Zumer ${ }^{\mathrm{c}}$, Daniel D. Wong ${ }^{\mathrm{d}}$, Sviatlana Dubovik ${ }^{\mathrm{a}}$, \\ Christoph M. Michel ${ }^{\mathrm{e}, \mathrm{f}}$, Armin Schnider ${ }^{\mathrm{a}}$ \\ ${ }^{a}$ Division of Neurorehabilitation, Department of Clinical Neurosciences, University Hospital Geneva, Avenue de Beau-Séjour 26, 1211 \\ Geneva, Switzerland \\ ${ }^{b}$ INSERM U1028, CNRS UMR5292, Lyon Neuroscience Research Center, Brain Dynamics and Cognition Team, 69675 Lyon, France \\ ${ }^{c}$ Sir Peter Mansfield Magnetic Resonance Centre, Physics and Astronomy, University of Nottingham, Nottingham, NG7 2RD, UK \\ ${ }^{d}$ Institute of Biomaterials and Biomedical Engineering, University of Toronto, Toronto, ON M5S3G9, Canada \\ ${ }^{e}$ Department of Fundamental Neuroscience, University of Geneva, 1211 Geneva, Switzerland \\ ${ }^{f}$ Department of Neurology, University of Geneva, 1211 Geneva, Switzerland
}

\begin{abstract}
BACKGROUND: The analysis of coherent networks from continuous recordings of neural activity with functional MRI or magnetoencephalography has provided important new insights into brain physiology and pathology. Here we assess whether valid localizations of coherent cortical networks can also be obtained from high-resolution electroencephalography (EEG) recordings.

METHODS: EEG was recorded from healthy subjects and from patients with ischemic brain lesions during a tonic hand muscle contraction task and during continuous visual stimulation with an alternating checkerboard. These tasks induce oscillations in the primary hand motor area or in the primary visual cortex, respectively, which are coherent with extracerebral signals (hand muscle electromyogram or visual stimulation frequency). Cortical oscillations were reconstructed with different inverse solutions and the coherence between oscillations at each cortical voxel and the extracerebral signals was calculated. Moreover, simulations of coherent point sources were performed.

RESULTS: Cortico-muscular coherence was correctly localized to the primary hand motor area and the steady-state visual evoked potentials to the primary visual cortex in all subjects and patients. Sophisticated head models tended to yield better localization accuracy than a single sphere model. A Minimum Variance Beamformer (MVBF) provided more accurate and focal localizations of simulated point sources than an $\mathrm{L}_{2}$ Minimum Norm (MN) inverse solution. In the real datasets, the MN maps had less localization error but were less focal than MVBF maps.
\end{abstract}

CONCLUSIONS: EEG can localize coherent cortical networks with sufficient accuracy.

Keywords: EEG, beamformer, minimum-norm, functional connectivity, imaginary coherence

${ }^{*}$ Correspondence should be addressed to:

Dr. Adrian Guggisberg

Tel: +41223823521

Fax: +41223823644

E-mail: aguggis@gmail.com 


\section{Introduction}

The brain is a complex network of dynamic systems with abundant functional interactions between local and more remote brain areas (Varela et al., 2001). Interregional neural communication is thought to be accompanied by a synchronization of oscillations between different brain regions (Aertsen et al., 1989; Engel et al., 1992; Gray et al., 1989; Gray and Singer, 1989). This interregional synchronization can be quantified with the concept of "functional connectivity" which is a measure of similarity between activity in different brain regions and is therefore considered to be an index of functional interaction (Gross et al., 2001; Lachaux et al., 1999; Nolte et al., 2004; Nunez et al., 1997; Stam et al., 2003). Studies using functional magnetic resonance imaging (fMRI) in healthy humans have shown that spontaneous fluctuations of brain activity at rest are highly organized and coherent within specific neuro-anatomical systems (Fox et al., 2005; Fransson, 2005). Thus, a careful analysis of coherence between brain regions gives access to the functional brain organization. Furthermore, the pattern of coherence between brain regions observed with fMRI at rest corresponds to the pattern of brain activation induced by corresponding tasks (Vincent et al., 2007), thus showing that techniques relying on functional connectivity do not depend on a correct task participation of the patients. Functional connectivity therefore opens an exciting and accessible window for a non-invasive assessment of brain function.

The concept of functional connectivity can be applied to any measurement of brain activity, such as fMRI, magnetoencephalography (MEG), or electroencephalography (EEG). EEG was the first brain signal to be used for calculating functional connectivity (Lopes da Silva et al., 1973; Thatcher et al., 1986). However, like MEG, it has the disadvantage that the indices of functional interaction calculated between sensors cannot be attributed to brain regions of interest or to structural lesions. Field spread leads to a wide representation of sources in many sensors, which makes the interpretation of functional connectivity measures between sensor pairs difficult (Schoffelen and Gross, 2009; Srinivasan et al., 2007).

For MEG, it has been shown that this limitation can be overcome by combining measures of functional connectivity with source localization algorithms. For instance, such "functional connectivity maps" (FCMs) can correctly localize the cortical generators of cortico-muscular interaction to the primary motor cortex (Gross et al., 2001; Jerbi et al., 2007; Schoffelen et al., 2008). We have shown that MEG maps of resting state intracortical interaction can correctly localize dysfunctional cortex in patients with brain tumors and distinguish it from tumor-infiltrated areas that are still functional (Guggisberg et al., 2008a; Martino et al., 2011).

Given the limited availability and high costs of MEG, it would be desirable to use source localization combined with functional connectivity measurements also for EEG recordings (Astolfi et al., 2005; De Vico Fallani et al., 2007). In principle, the algorithms used in MEG studies can be applied also to EEG, but there are differences between EEG and MEG signals that need to be considered.

- Electric fields are distorted to a greater extent than magnetic fields by the inhomogeneous conductivities of the brain, skull, and scalp. EEG is therefore more dependent on accurate head models to resolve the forward problem than MEG. The analysis of patients with brain lesions could also be problematic because of different conductivity of lesion tissue compared to healthy tissue.

- When functional connectivity is assessed between EEG sensors, the choice of the reference electrode critically influences and potentially biases the results (Fein et al., 1988; Guevara et al., 2005). Projecting EEG sensor data through inverse solutions to the brain effectively eliminates the reference problem of EEG studies, since the data becomes reference-free (Fender, 1987; Geselowitz, 1998). However, previous studies have reported that the choice of the reference electrode can lead to minor variations in the output of minimum variance beamformers (MVBF) (Van Veen et al., 1997).

This study aimed to evaluate the feasibility of localizing cortico-peripheral coherence with EEG in healthy subjects and patients with brain lesions, and to compare the performance of different analysis parameters. More specifically, we assessed the influence of the head model, the inverse solution, the method for determination of dipole orientations, and (for beamformers) the reference electrode on the accuracy of functional connectivity localization.

To this end, we collected two sets of real data in which cortical nodes with known location oscillate in synchrony with extracerebral activity.

- Tonic muscle contraction is known to induce beta frequency $(\sim 15-30 \mathrm{~Hz})$ oscillations in the contralateral primary motor cortex which are coherent with coregistered electromyographic (EMG) activity (Mima and Hallett, 1999; Murthy and Fetz, 1992). Hence, the algorithms should localize cortical activity that is coherent with EMG activity to the contralateral primary motor cortex.

- Rhythmic visual stimulation is known to induce a steady state visual evoked potential (SSVEP) in the primary visual cortex, which oscillates in the frequency of the stimulation and harmonics (Bianciardi et al., 2009; Emir et al., 2008; Victor et al., 1994). Hence, the algorithms should localize cortical oscillations that are coherent with the stimulation frequency to the primary visual cortex.

In order to test whether the EEG localizations remain valid in patients with brain lesions, we obtained the data not 
only from healthy subjects but also from patients with unilateral territorial ischemic stroke.

In addition, we carried out simulations of point sources to evaluate the performance of different inverse solutions.

\section{Methods}

\subsection{Subjects and patients}

Two healthy subjects and four patients with ischemic stroke participated in this study (mean age 44, age range 29-70, 3 women). The lesions were located in the territory of the left middle cerebral artery in 3 patients and of the right anterior cerebral artery in one patient (Figures 1 and 2 , blue arrows).

\subsection{Recordings}

Recordings were obtained with a 128-channel Biosemi ActiveTwo system (Biosemi V.O.F. Amsterdam, Netherlands), using a sampling rate of $512 \mathrm{~Hz}$. Impedances were kept below $5 \mathrm{k} \Omega$.

Bad EEG channels as determined by visual inspection were excluded. The data was re-referenced to the average of all non-excluded channels. We additionally created a second version of EEG data by re-referencing to the vertex electrode $(\mathrm{Cz})$, in order to assess the influence of the reference montage on beamformers (see below).

\subsection{Corticomuscular coherence task}

EEG and EMG of the first dorsal interosseus (FDI) muscle were recorded simultaneously in 1 healthy subject and 3 patients during a tonic muscle contraction task. The contractions were performed with the non-paretic hand ipsilateral to the lesioned hemisphere in 2 patients with hemiparesis, and with the hand contralateral to the lesioned hemisphere in one patient without hemiparesis. Bipolar surface EMG was recorded at $512 \mathrm{~Hz}$ with the active EMG electrode fixed on the FDI muscle belly and the reference electrode $\sim 4 \mathrm{~cm}$ proximal over the volar part of the radius head. The participants were visually instructed by a hand symbol displayed on a monitor to exert about $50 \%$ of their maximum force during repeated periods of $10 \mathrm{~s}$ which were separated by pauses of $5 \mathrm{~s}$. The EEG and EMG data were high-pass filtered at $10 \mathrm{~Hz}$ with a fifth-order ellipsoid IIR filter. Forward and backward filtering was applied to obtain zero-phase distortion. Segments with artifacts as well as the resting periods were excluded. A total of at least $3 \mathrm{~min}$ of artifact-free EEG and EMG signal during FDI contraction were obtained for each participant.

\subsection{Steady state visual evoked potential (SSVEP) task}

EEG recordings during continuous visual stimulation with a bilateral, alternating, black and white checkerboard pattern were obtained in 2 healthy subjects and 2 patients with ischemic stroke. All patients had a normal visual field as determined clinically. Participants were asked to
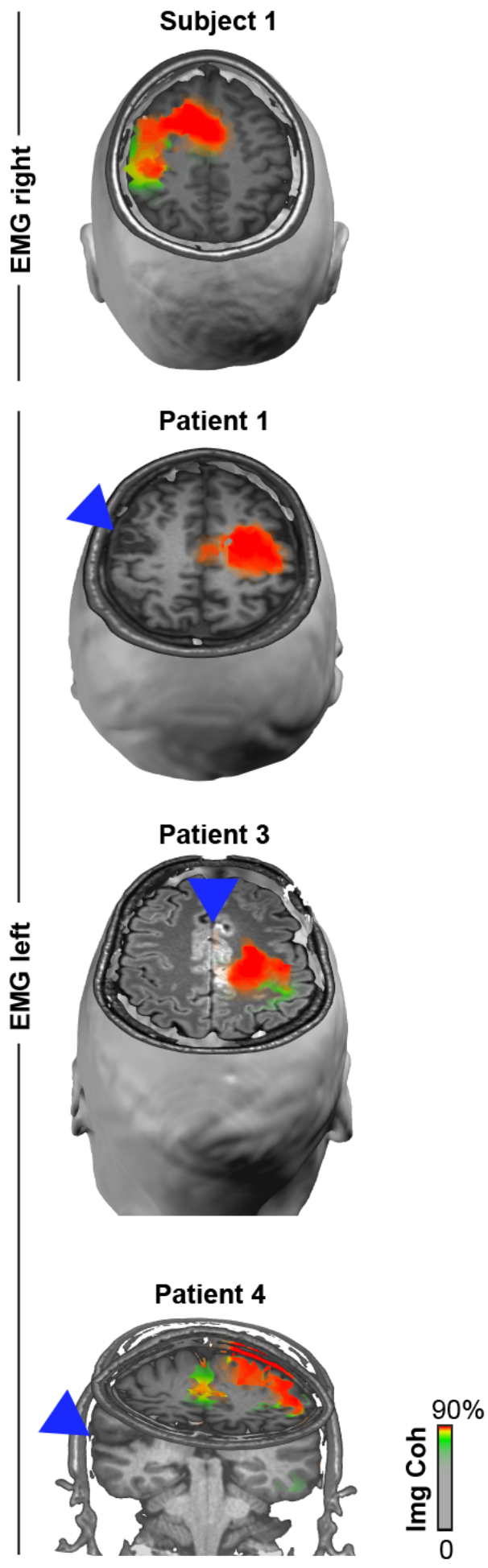

Figure 1: Functional connectivity maps (FCMs) of corticomuscular coherence in 1 healthy subject and 3 patients with ischemic brain lesions (marked with blue arrows). The EMG was recorded from an intrinsic hand muscle (first dorsal interosseus). The imaginary component of corticomuscular beta-band coherence (IC) is indicated as $\%$ of the individual maximum value at the peak voxel. The peak absolute imaginary coherence magnitude was $0.11 \pm 0.02($ mean $\pm \mathrm{SD})$. $\mathrm{IC}$ was greatest around the hand notch in all participants. Average referenced EEG recordings, a SMAC head model, dipole orientations with maximum output SNR, and a Minimum Norm inverse solution were used for all maps. 


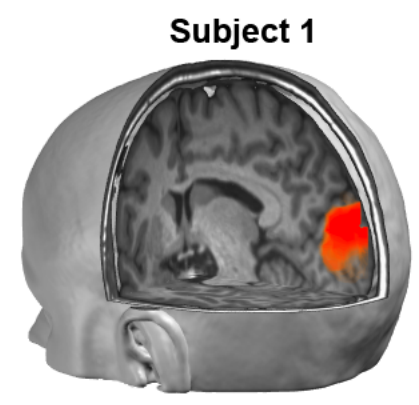

Subject 2

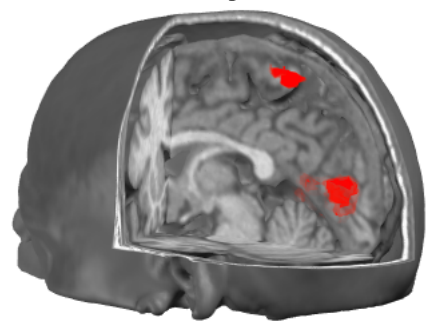

Patient 2

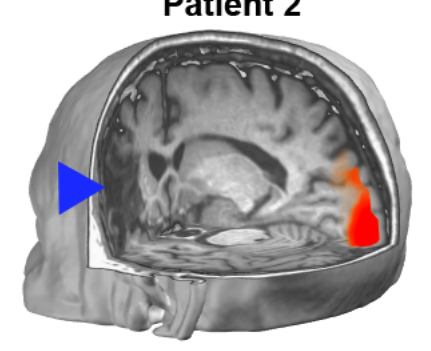

Patient 3
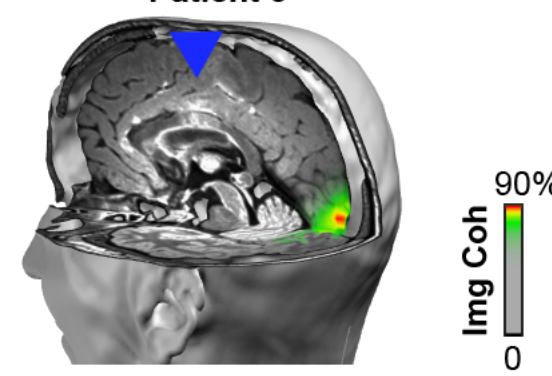

Figure 2: FCMs of steady-state visual evoked potentials (SSVEP) oscillating coherently with the visual stimulation frequency obtained from 2 healthy subjects and 2 patients with ischemic brain lesions (marked with blue arrows). The IC is indicated as \% of the individual maximum value at the peak voxel. The peak absolute imaginary coherence magnitude was $0.49 \pm 0.07$ (mean \pm SD). IC at the stimulation frequency was largest around the primary visual cortex in all participants. The same analysis parameters as in Figure 1 were used. fixate a red cross in the center of the screen. The interval between the alternating patterns was set to $200 \mathrm{~ms}$, which corresponds to an alternation frequency of $5 \mathrm{~Hz}$. The EEG was (forward and backward) band-pass filtered between 4 and $6 \mathrm{~Hz}$ with a fifth-order ellipsoid IIR filter. Segments with artifacts were rejected by visual inspection. A total of at least 3 min of artifact-free EEG signal during visual stimulation were obtained for each subject. In addition, a $5 \mathrm{~Hz}$ sinusoidal oscillation representing the visual stimulation was created.

\subsection{Structural imaging}

A high-resolution, T1-weighted cerebral MRI covering the entire skull was obtained for each participant. In addition, a MNI standard volume consisting of the average of 152 healthy subjects was used for coregistration in order to assess the impact on localization accuracy.

\subsection{Head models and lead-potential}

Each subject's brain was segmented into scalp, skull, gray and white matter with the software BrainSuite09 (http://www.loni.ucla.edu/ shattuck/ brainsuite/) written by David Shattuck. The gray matter volume was divided into a regular $10 \mathrm{~mm}$ grid. Three different head models were used to calculate the leadpotential for each grid location.

\section{Spherical model with anatomical constraints (SMAC)}

The SMAC head models were created with the software SMAC Toolbox (Functional Brain Mapping Laboratory, University of Geneva) written by Franois Tavel. The algorithm of this head model is described elsewhere (Spinelli et al., 2000). In short, the individual T1 MRI was transformed to a best-fitting sphere. The solution points were defined in the gray matter tissue of this deformed MRI and the lead field was computed using an analytical solution for a 3-shell spherical head model (Ary et al., 1981) which assumes conductivities of 1:0.0125:1 and radii of 1:0.92:0.87 for scalp, skull, and brain, respectively (Rush and Driscoll, 1968).

\section{Berg 3-shell single-sphere head model}

This model was created with the NUTEEG plugin (Wong and Gordon, 2009) of NUTMEG (http://nutmeg. berkeley.edu) (Dalal et al., 2011). A single sphere with the best fit to the individual skull shape was defined and 3 concentric shells with radii of 1:0.93:0.85 relative to the fitted sphere were created (Berg and Scherg, 1994). The solution points were confined to the gray matter as defined by the segmented MRI of the subject. The conductivities for scalp, skull, and brain, were set to the same standard values as for SMAC. 


\section{Boundary Element Head Model}

A 3-shell BEM model was created with the Helsinki BEM library (http://peili.hut.fi/BEM/) (Stenroos et al., 2007) and the NUTEEG plugin of NUTMEG. The BEM approach constructs a realistic head model by defining discrete 3-D compartments comprising the brain, scalp, and skull. Each compartment is assumed to have uniform electrical conductivity. Together with the shape of each compartment, this information is then used to compute the voltage at the electrode positions expected to result from a source with a given position and orientation (Mosher et al., 1999). We created triangular meshes for scalp, and outer and inner skull surface based on the segmented T1 MRI (with 1000, 1500, and 2000 nodes, respectively). The lead-potential was calculated over the grid of gray matter solution points. The conductivities for each shell were set identically to the SMAC and Berg models.

The electrode positions were determined by fitting and projecting the spherical standard electrode coordinates to the individual scalp form.

\subsection{Scalarization of the lead-potential}

For the localization of functional connectivity in the brain, we require an estimation of neural network oscillations at each solution point. Vector weights obtained from the inverse solution allow only reconstructing squared power values for a given location; the reconstruction of neural oscillations requires a scalar weight matrix. In order to scalarize the lead-potential as input to scalar weights computation, we need to determine the dipole orientation at each grid location. Here, we compared two different techniques to achieve this.

(i) Computation of an optimal dipole orientation yielding maximum output SNR. For the MVBF, the optimum orientation at each dipole $\boldsymbol{r}$ is given by (Sekihara et al., 2004):

$$
\boldsymbol{\eta}_{\text {opt }}(\boldsymbol{r})=\boldsymbol{v}_{\min }\left\{\left[\boldsymbol{L}^{T}(\boldsymbol{r}) \boldsymbol{R}^{-1} \boldsymbol{L}(\boldsymbol{r})\right]^{-1}\left[\boldsymbol{L}^{T}(\boldsymbol{r}) \boldsymbol{R}^{-2} \boldsymbol{L}(\boldsymbol{r})\right]\right\}
$$

where $\boldsymbol{v}_{\min }$ is the eigenvector corresponding to the minimum eigenvalues of the matrix in \{\}$, \boldsymbol{L}$ is the vector leadpotential, $\boldsymbol{R}$ the sensor covariance matrix, and superscript $T$ denotes the matrix transpose.

For the MN inverse solution, it is obtained as (PascualMarqui et al., 2009):

$$
\boldsymbol{\eta}_{\text {opt }}(\boldsymbol{r})=\boldsymbol{v}_{\max }\left\{\boldsymbol{L}^{T}(\boldsymbol{r}) \boldsymbol{G}^{-1} \boldsymbol{R} \boldsymbol{G}^{-1} \boldsymbol{L}(\boldsymbol{r})\right\},
$$

where $\boldsymbol{v}_{\max }$ the eigenvector corresponding to the maximum eigenvalues of the matrix in \{\} , and $\boldsymbol{G}=\boldsymbol{L} \boldsymbol{L}^{T}$ is the Gram matrix.

(ii) Anatomically constrained surface normal dipole orientations. A model of the individual cortical surface was created for each participant with BrainSuite09. The number of vertices of the cortical surface model was downsampled to 8000 . For each solution point, the dipole orientation was defined as normal to the orientation of the closest cortex surface with the NUTEEG plugin of NUTMEG.

The scalar lead-potential was then calculated as:

$$
\boldsymbol{l}\left(\boldsymbol{r}, \boldsymbol{\eta}_{o p t}\right)=\boldsymbol{L}(\boldsymbol{r}) \boldsymbol{\eta}_{o p t}(\boldsymbol{r})
$$

and will henceforth be designated $\boldsymbol{l}(\boldsymbol{r})$ for simplicity.

\subsection{Inverse solutions}

Inverse solutions were calculated with NUTMEG (Dalal et al., 2011). A spatial filter matrix $\boldsymbol{w}$ was calculated for estimation of the signal at each grid location. Two different inverse solutions were compared. The minimum variance beamformer (MVBF) (Van Veen et al., 1997; Vrba and Robinson, 2001; Sekihara et al., 2001, 2005 ) is an adaptive spatial filter which uses the sensor covariance matrix $\boldsymbol{R}$ of the entire recording to estimate the spatial filter matrix $\boldsymbol{w}$.

$$
\boldsymbol{w}_{M V B F}(\boldsymbol{r})=\frac{\boldsymbol{R}^{-1} \boldsymbol{l}(\boldsymbol{r})}{\boldsymbol{l}^{T}(\boldsymbol{r}) \boldsymbol{R}^{-1} \boldsymbol{l}(\boldsymbol{r})}
$$

For our average-referenced EEG datasets, we used the Moore-Penrose pseudoinverse to estimate $\boldsymbol{R}^{-1}$ for the following reason. An average reference introduces a linear dependence between the channels, such that any given channel can be calculated as a sum of the remaining channels, reducing the rank of the data matrix by exactly 1 . The sensor covariance matrix then becomes rank-deficient and invertible only with regularized inversion methods. An alternative solution to this problem is to apply a rotation to the rank-deficient covariance matrix such that the last column contains zeros, and the remaining columns contain independent data. The same rotation is then also applied to the lead-potential, to keep the contributions of each channel identical. Both the pseudoinverse and the matrix rotation approach produced identical beamformer output for the tested data.

The covariance can also become ill-conditioned when using especially short data lengths or bandwidths, in which case Tikhonov regularization procedures can be applied (Brookes et al., 2008). This was however not the case in our datasets.

In order to ensure that the MVBF performance was not sensitive to the choice of the data bandwidth used for calculation of the covariance matrix, we compared three different bandwidths for the analysis of corticomuscular coherence in 2 subjects. One subject had the individual corticomuscular coherence peak at $24 \mathrm{~Hz}$, the second subject at $26 \mathrm{~Hz}$. The frequency bands $>10 \mathrm{~Hz}, 20-$ $30 \mathrm{~Hz}$, and peak coherence $\pm 1 \mathrm{~Hz}$ were compared. The resulting FCMs were highly correlated among each other $(r>.78)$, and the coherence peaks located within the same $10 \mathrm{~mm}$ radius. For the main analyses of the corticomuscular dataset, the sensor covariance was calculated from the data that was high-pass filtered at $10 \mathrm{~Hz}$. A bandwidth of 4 to $6 \mathrm{~Hz}$ was used for the SSVEP dataset. 
An alternative version of a beamformer is "dynamic imaging of coherent sources (DICS)" (Gross et al., 2001). DICS uses correlations in the frequency domain (a crossspectrum density matrix) for source estimation instead of temporal correlations (sensor covariance matrix) used for the MVBF. Given the similarity of the two approaches, DICS was not separately tested in this study.

The $\mathrm{L}_{2}$ Minimum Norm (MN) is a non-adaptive inverse solution which tries to minimize the overall $3-\mathrm{D}$ current distribution. We used the basic MN algorithm (Hämäläinen and Ilmoniemi, 1994):

$$
\boldsymbol{w}_{M N}(\boldsymbol{r})=\frac{\boldsymbol{l}(\boldsymbol{r})}{\boldsymbol{l}^{T} \boldsymbol{l}+\lambda \boldsymbol{I}},
$$

where $\lambda \boldsymbol{I}$ is a regularization matrix.

Weighted versions of $\mathrm{MN}(\mathrm{WMN}$ ) additionally allow to re-weight the inverse solution, e.g., with regards to the depth of the solutions points or with regards to prior knowledge about the spatial distribution of the source activity (Dale et al., 2000; Lin et al., 2004). These variants are not evaluated here.

The inverse solutions sLORETA (Pascual-Marqui, 2002) and dSPM (Dale et al., 2000) additionally standardize the MN or WMN solution by the variance of the estimated current density $\boldsymbol{J}$ :

$$
\boldsymbol{w}_{s M N}(\boldsymbol{r})=\boldsymbol{w}_{M N}(\boldsymbol{r}) \boldsymbol{J}(\boldsymbol{r})
$$

We show below that the standardization $\boldsymbol{J}$ is canceled out during calculation of source space coherence. The sLORETA and dSPM algorithms therefore yield the same source coherence results as (W)MN algorithms.

\subsection{Functional connectivity in source space}

Localization of coherence in source space was done with the FCM plugin of NUTMEG written by AGG. The EEG, EMG and visual stimulation sinusoid were divided into non-overlapping data segments of $1 \mathrm{~s}$ duration (512 data points). A fast Fourier transform (FFT) was performed for each data segment and sensor. For the corticomuscular coherence, a multi-taper approach was used (Mitra and Pesaran, 1999). Each data segment was tapered using a set of discrete prolate spheroidal sequences (Slepian functions). The number of tapers used determines the amount of spectral smoothing. As described previously (Schoffelen et al., 2008), we maximized the sensitivity of subsequent analysis steps by determining the peak frequency and the amount of spectral smoothing individually for each subject. To this end, we computed the coherence between the EMG and the contralateral central electrode (C3 or C4) using different numbers of tapers. We then selected the number of tapers which produced a coherence peak with a width corresponding approximately to the smoothing bandwidth. The frequency was set at the coherence peak. For the SSVEP dataset, the Fourier coefficients were calculated for the frequency band between 4 and $6 \mathrm{~Hz}$.
The complex Fourier coefficients $\boldsymbol{F}$ of all electrodes at frequency $f$ were then projected through the spatial filter $\boldsymbol{w}$ into the source space:

$$
\boldsymbol{d}(\boldsymbol{r}, f)=\boldsymbol{w}^{T}(\boldsymbol{r}) \boldsymbol{F}(f)
$$

This is equivalent to first projecting the filtered data to source space and then calculating the FFT at each grid location (Guggisberg et al., 2008a), but is computationally more efficient.

The complex coherence between neural oscillations at each grid location and either the EMG or the visual stimulus sinusoid could then be computed as

$$
C C(\boldsymbol{r}, f)=\frac{\sum_{k=1}^{K} \boldsymbol{d}_{k}(\boldsymbol{r}, f)^{*} \boldsymbol{E}_{k}(f)}{\sqrt{\sum_{k=1}^{K}\left|\boldsymbol{d}_{k}(\boldsymbol{r}, f)\right|^{2} \sum_{k=1}^{K}\left|\boldsymbol{E}_{k}(f)\right|^{2}}}
$$

where $k=[1, \ldots, K]$ stands for the data segments, * for the complex conjugate, and $\boldsymbol{E}$ for the Fourier transformed extracerebral signals (EMG or visual stimulus sinusoid), averaged (in the case of EMG) across all tapers.

From the complex coherence, the absolute imaginary component (IC) (Nolte et al., 2004) as well as the magnitude squared coherence (MSC) were computed:

$$
\begin{gathered}
I C=|\Im\{C C\}| \\
M S C=|\{C C\}|^{2}
\end{gathered}
$$

When calculating cortico-peripheral coherence with sLORETA or dSPM, we obtain from equations 6,7 and 8 above:

$$
\begin{gathered}
C C(\boldsymbol{r}, f)=\frac{\sum_{k=1}^{K} \boldsymbol{w}_{M N}(\boldsymbol{r}) \boldsymbol{J}(\boldsymbol{r}) \boldsymbol{F}_{k}(f)^{*} \boldsymbol{E}_{k}(f)}{\sqrt{\sum_{k=1}^{K}\left|\boldsymbol{w}_{M N}(\boldsymbol{r}) \boldsymbol{J}(\boldsymbol{r}) \boldsymbol{F}_{k}(f)\right|^{2} \sum_{k=1}^{K}\left|\boldsymbol{E}_{k}(f)\right|^{2}}} \\
=\frac{\boldsymbol{J}(\boldsymbol{r})}{\sqrt{|\boldsymbol{J}(\boldsymbol{r})|^{2}}} \frac{\sum_{k=1}^{K} \boldsymbol{w}_{M N}(\boldsymbol{r}) \boldsymbol{F}_{k}(f)^{*} \boldsymbol{E}_{k}(f)}{\sqrt{\sum_{k=1}^{K}\left|\boldsymbol{w}_{M N}(\boldsymbol{r}) \boldsymbol{F}_{k}(f)\right|^{2} \sum_{k=1}^{K}\left|\boldsymbol{E}_{k}(f)\right|^{2}}}
\end{gathered}
$$

Since the elements of $\boldsymbol{J}$ are positive real, $\boldsymbol{J}$ is canceled out during calculation of cortico-peripheral coherence. The same can be shown for calculations of corticocortical coherence. Hence, standardized (W)MN variants such as sLORETA and dSPM do not provide additional advantage over (W)MN for the localization of coherence.

\subsection{Visualization}

3-D renderings of the FCMs were performed with Cartool (Brunet et al., 2011) (http://sites.google.com/ site/cartoolcommunity/home). 


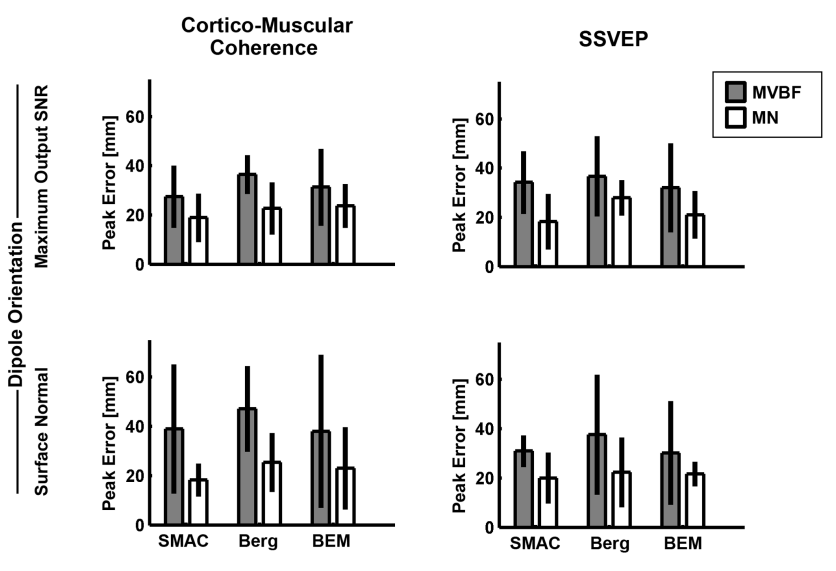

Figure 3: Comparison of the performance of different analysis parameters for FCMs of the imaginary component of coherence (IC). The distance between the FCM peak and the anatomically defined target is indicated. Note that the Minimum Norm inverse solution (MN) provided significantly better localization accuracy than a Minimum Variance Beamformer (MVBF). The SMAC and BEM head models showed less localization error than a Berg single sphere head model, although the difference did not reach statistical significance. The method for calculating dipole orientations did not significantly influence the localization accuracy.

\subsection{Evaluation of Performance}

The anatomical centers of the hand notch and of the interhemispheric junction of the calcarian sulcus were defined individually in the co-registered MRIs. The distance between the FCM peak coherence and these anatomical targets were used as an index of FCM accuracy.

\subsection{Simulations}

We simulated single cortical point sources with a continuous $10 \mathrm{~Hz}$ sinusoidal rhythm while the remaining cortical grid locations were set to Gaussian random oscillations. An additional extracerebral $10 \mathrm{~Hz}$ oscillation was defined with a radial phase lag of $\pi / 2(=25 \mathrm{~ms})$ relative to the cortical point source. This phase difference leads to maximal values in the imaginary part of coherence. The dipole orientations were fixed to be normal to the cortical surface. The cortical sources were then projected to the EEG sensors by using a scalar lead-potential calculated with a SMAC head model. Four different levels of Gaussian white random noise were added to the sensors (SNR 1, 2, 5, or 10). The simulated sensor data was then bandpass filtered between 1 and $20 \mathrm{~Hz}$, and projected back to all gray matter grid locations through a spatial filter matrix calculated with the MVBF and MN inverse solutions described above. The IC between the extracerebral sinusoid and all cortical grid locations was then computed for the $10 \mathrm{~Hz}$ frequency bin. The distance between the IC peak and the source coordinate was then calculated to determine the localization error. The spatial spread of the obtained maps was quantified as the full-width-half-maximum (FWHM) around the IC peak. The sources were placed randomly in the cortex of healthy subject 1 . The results of 20 different locations distributed throughout the entire cortex were averaged.

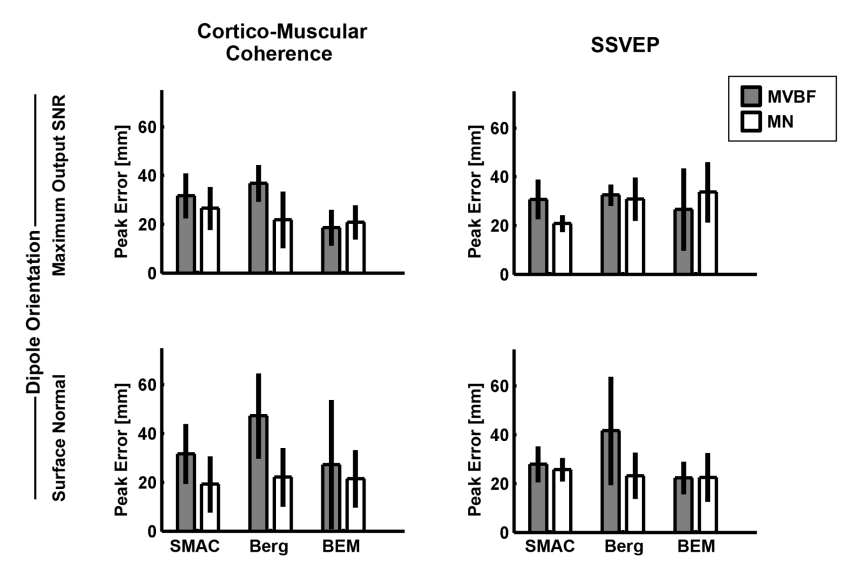

Figure 4: Comparison of the performance of different analysis parameters for FCMs of magnitude squared coherence (MSC). The Minimum Norm inverse solution (MN) provided better localization accuracy than a Minimum Variance Beamformer (MVBF). The SMAC and BEM head models showed less localization error than a Berg single sphere head model, although the difference did not reach statistical significance. The method for calculating dipole orientations did not significantly influence the localization accuracy.

\subsection{Statistics}

A three-way analysis of variance (ANOVA) was used to compare the peak-to-peak error and FWHM of FCM maps obtained with different head models (SMAC vs. single sphere vs. BEM), methods for determining the dipole orientation (maximum output SNR vs. surface normal), and inverse solutions (MVBF vs. MN).

\section{Results}

\subsection{Real Data}

Figures 1 and 2 demonstrate that EEG-based FCMs correctly localized the imaginary component of corticomuscular coherence to the primary motor cortex and SSVEP oscillations to the primary visual cortex in all subjects and in all patients with brain lesions.

Figure 3 visualizes the peak-to-target error of FCMs obtained with different analysis parameters. The choice of the inverse solution had a significant impact on the accuracy of imaginary coherence localizations, with MN performing significantly better than the MVBF, both in the cortico-muscular coherence (overall mean $\pm \mathrm{SD}: \mathrm{MN}$, $21 \pm 4 \mathrm{~mm}$; MVBF, $\left.37 \pm 12 \mathrm{~mm} ; F_{1,47}=10.2, \mathrm{p}=0.0028\right)$ as well as in the SSVEP dataset (MN, $22 \pm 7 \mathrm{~mm}$; MVBF, $\left.34 \pm 14 \mathrm{~mm} ; F_{1,47}=8.6, \mathrm{p}=0.0057\right)$. On the other hand, MVBF produced maps with less spatial spread than MN in both the corticomuscular coherence (44 vs. $65 \mathrm{~mm}$, $\left.F_{1,47}=10.5, \mathrm{p}=0.0025\right)$ and the SSVEP dataset (36 vs. $\left.46 \mathrm{~mm}, F_{1,47}=3.8, \mathrm{p}=0.060\right)$. The choice of the head model and of the method for determining the dipole orientation did not significantly affect the localization accuracy in either dataset $(\mathrm{p} \geq 0.28)$, although Figure 3 shows a nonsignificant difference in performance in favour of SMAC and BEM head models as compared to a single Berg sphere 
(overall mean of both datasets and both inverse solutions: SMAC, 26 $\pm 3 \mathrm{~mm}$; Berg, $32 \pm 8 \mathrm{~mm}$; BEM, $27 \pm 3 \mathrm{~mm}$ ). With an optimal choice of parameters (SMAC or BEM head model, MN inverse solution), the peak-to-target localization error was $<20 \mathrm{~mm}$ for both datasets.

When comparing average-referenced to vertex $(\mathrm{Cz})$ referenced sensor data, we found a non-significant but nonvanishing difference in MVBF localization accuracy for either dataset $(\mathrm{p} \geq 0.33)$. The sources of this difference will be discussed below.

When a standard MNI template instead of the individual MRI was used for the creation of a SMAC head model, the peak error of localizations obtained with MN was larger for the corticomuscular coherence dataset $(27 \mathrm{~mm}$ instead of $19 \mathrm{~mm}$ ), but not for the SSVEP dataset $(18 \mathrm{~mm}$ in both cases).

The performance of MSC maps is shown in Figure 4, and was not significantly different from the performance of imaginary coherence maps $\left(F_{1,95}=0.58, \mathrm{p}=0.45\right)$. Again, the inverse solution had a significant influence on performance in both datasets $\left(F_{1,47} \geq 7.9, \mathrm{p} \leq 0.008\right)$, in favor of MN. The choice of the head model $\left(F_{1,47} \leq 2.5, \mathrm{p} \geq 0.1\right)$ and the method for determination of the dipole orientations $\left(F_{1,47} \leq 0.48, \mathrm{p} \geq 0.49\right)$ were not associated with significantly different localization errors.

\subsection{Simulations}

The MVBF localized the single point sources with significantly less error $\left(F_{1,159}=9.9, \mathrm{p}=0.002\right)$ and less spatial spread $\left(F_{1,159}=25.2, \mathrm{p}<0.0001\right)$ than MN (see Figure 5 ). As expected, the SNR only influenced the spatial spread of the MVBF $\left(F_{1,79}=29.2, \mathrm{p}<0.0001\right)$, but not of the MN solution $\left(F_{1,79}=0.32, \mathrm{p}=0.57\right)$

\section{Discussion}

This study demonstrates that coherent sources can be localized with reasonable accuracy from EEG data. With optimal analysis parameters, we observed a mean distance between the center of the anatomical target regions and the FCM peaks of $<20 \mathrm{~mm}$. Given that both FCMs and anatomical targets had extensions of $>20 \mathrm{~mm}$, this corresponds to a good overlap (shown in Figures 1 and 2), which is sufficient for localization of large-scale networks across the entire cortex. Hence, the analysis of coherent functional networks which has proven to offer exciting new possibilities in fMRI (Carter et al., 2010; He et al., 2007; Raichle and Snyder, 2007; Vincent et al., 2007) and MEG studies (Castellanos et al., 2010; de Pasquale et al., 2010; Guggisberg et al., 2008a; Jerbi et al., 2007) is also possible with EEG data. In comparison with fMRI, EEG has the disadvantage of lower spatial resolution, but the advantage that it can assess actual neural oscillations at different spectral frequencies. The localization of corticomuscular coherence, for example, would not be possible with fMRI. Furthermore, EEG recordings are less costly
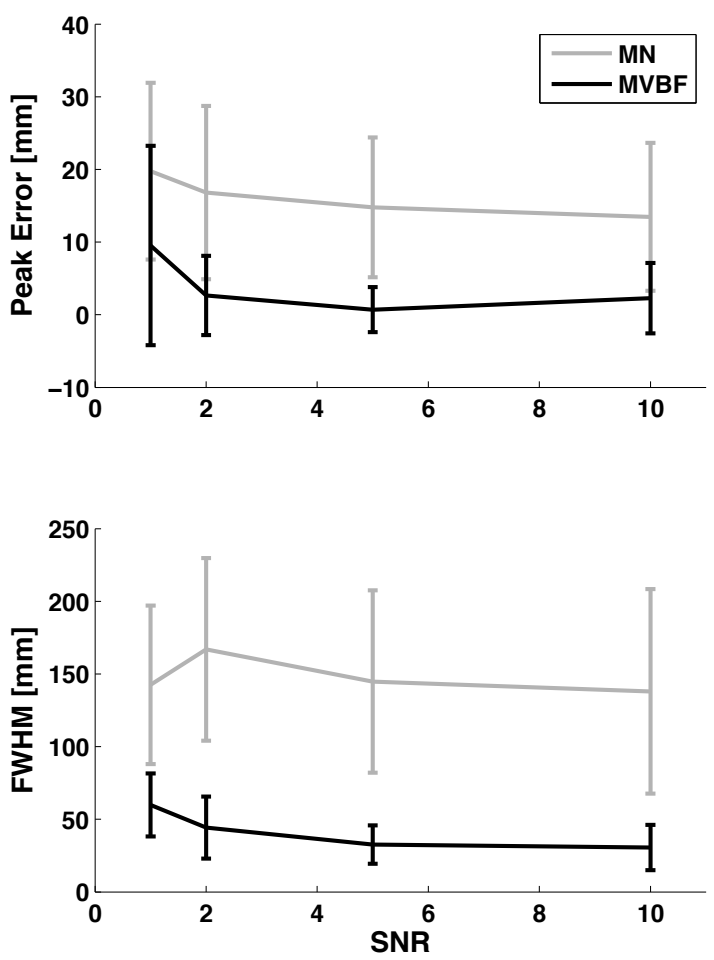

Figure 5: Simulations of single point sources oscillating in synchrony with an external sinusoid were performed. The graph shows the mean $( \pm \mathrm{SD})$ peak error and spatial spread (full-width-halfmaximum, FWHM) of 20 separately simulated source locations distributed randomly in the entire cortex of one healthy subject.

and more widely available than fMRI or MEG recordings. Unlike MEG, EEG may also be recorded simultaneously with fMRI.

A methodological limitation of our study is that we did not individually record the position of EEG electrodes, but merely fit standard coordinates to the individual head shape. The usage of digitized electrode positions may improve the localization accuracy reported here. However, the corresponding infrastructure is not generally available and our data shows that reasonably precise localizations can be obtained even with this approach.

\subsection{Inverse solutions}

The most important parameter for localization accuracy and distribution was the choice of the inverse solution. Non-adaptive algorithms provided significantly better localization accuracy in real datasets than adaptive algorithms. On the other hand, beamformers yielded more accurate localizations of point sources in the simulations. This apparent contradiction can be explained with the principle of operation of the inverse solutions. The MVBF uses the temporal covariance of the EEG data (in addition to the sensor geometry) to create a custom spatial filter depending on the signal characteristics. This en- 
ables more precise and focal source localizations (Sekihara et al., 2005; Dalal et al., 2008; Guggisberg et al., 2008b) that we also observe here in the simulations. However, beamformers are especially sensitive to the accuracy of the head model; measured data that is inconsistent with the head model is liable to be rejected as noise (Steinsträter et al., 2010). Additionally, the beamformer approach assumes that reconstructed sources are temporally uncorrelated with each other. In case of spatially distant sources that are correlated with each other and coherent with peripheral oscillations, the algorithm may therefore yield erroneous localizations (Hadjipapas et al., 2005; Sekihara et al., 2002; Van Veen et al., 1997). In continuous recordings of real data, such correlations may occur and seem to outweigh the technical advantages of MVBF for the localization of coherent functional networks. Yet, the performance differences observed in real data must be interpreted with caution, because we do not know the true location of strongest cortico-peripheral coherence in real datasets. For instance, there is evidence not only for involvement of the primary motor cortex in corticomuscular coherence but also of somatosensory and parietal areas (Witham et al., 2010; Baker, 2007). Premotor areas could also be involved. The peak of corticomuscular coherence could therefore be shifted away from the hand notch and lead to biased performance estimates of the inverse solutions. The peak coherence location estimated by the MVBF was on average anterior to the hand notch and to the localizations of MN. Hence, if the true corticomuscular coherence peak was anterior to the hand notch, this would reduce the inferior performance of MVBF compared to $\mathrm{MN}$ we observed. In any case, valid localizations of motor and visual coherent sources could be achieved in all participants also with MVBF, although the distance to the anatomical target used here was $\sim 10 \mathrm{~mm}$ larger than with MN.

The spatial spread of FCMs has similar characteristics as the spatial spread of activation maps and follows the point-spread function of each algorithm (Sekihara et al., 2005). The MVBF provides significantly more focal localizations than MN both in simulations and real data.

Although to our knowledge no systematic comparison of adaptive vs. non-adaptive algorithms has been performed for FCMs from MEG data so far, it is probable that the differences found with EEG data in this study also apply to MEG data.

\subsection{EEG-specific parameters}

The parameters that are specific for EEG studies turned out to be of relatively minor importance for localization accuracy of coherence. We did not observe significant differences in performance between the different head models, and valid results could even be obtained without a co-registered individual structural MRI with head models based on MRI templates. However more precise head models (SMAC and BEM) yielded better localization accuracy, and should therefore be preferred. The motor and visual cortex that were used as targets in this study are relatively superficial; the accuracy of the head model is probably more critical for the localization of deeper networks, e.g., in the hippocampus. Furthermore, studies using a larger number of participants and a smaller solution grid size will probably find significant performance differences even for superficial sources. Future studies could address the effect of different head models on coherence localisation with simulations, e.g., by creating simulations with a finite element model (FEM) and by evaluating the performance of other head models such as the ones used in this manuscript.

Valid localizations of coherent cortical sources were not only obtained in healthy participants but also in all patients with ischemic brain lesions. Hence, it is feasible to use the EEG to map functional connectivity also in patients with brain lesions. However, our study was not designed to make a systematic comparison between patients and healthy controls, and does therefore not rule out a performance decrease in patients, especially in areas close to the lesions.

In sharp contrast with functional connectivity analyses between EEG sensors where the choice of the reference is crucial (Fein et al., 1988; Guevara et al., 2005), the reference montage has no significant influence on localization accuracy of source space connectivity maps, since the inverse solutions make the data reference-free (Fender, 1987; Geselowitz, 1998). However, in accordance with a previous report (Van Veen et al., 1997), we found non-vanishing differences between average- and vertex-referenced montages when using a beamformer. This may be initially surprising since the coordinate transform introduced by the reference is applied equally to the lead-potential and the data and therefore should be canceled. However, the observed differences arise for 2 reasons. First, the inversion of the sensor covariance matrix $\boldsymbol{R}$ introduces minor variances leading to reference dependent variations in the localizations. This can be avoided by using the pseudoinverse of $\boldsymbol{R}$ (or other constant regularization across the different reference choices), even when the matrix is well-conditioned, which yields reference-independent results for vector beamformers. Second, equation (1) used for determination of the dipole orientation with maximum SNR for the MVBF is not neutral to a coordinate transform introduced by the EEG reference and therefore explains the non-vanishing differences observed here with a scalar beamformer. Nevertheless, the differences are minor and did not reach significance in our datasets $(\mathrm{p} \geq 0.33)$. By the way, the $\mathrm{MN}$ algorithm for calculation of dipole orientation used here (equation 2) is reference-independent.

\subsection{Dipole orientation}

Concerning dipole orientations, our results show that output maximization algorithms yield similar FCM performance as anatomically defined surface normal orientations, while being more convenient and requiring much 
less human work load. This is in agreement with a previous MEG study which showed that surface normal dipole orientations are only advantageous if the MEG-MRI coregistration error is smaller than $2 \mathrm{~mm}$ and the error in the estimation of the cortical surface orientation is smaller than $10^{\circ}$ (Hillebrand and Barnes, 2003). Such output maximization algorithms are valid not only for beamformers but also for non-adaptive algorithms. We can, however, not rule out minor performance differences which might have been masked due to the relatively low number of participants in this study. Future studies could use simulations of different dipole orientations to compare more systematically the performance of the two procedures for orientation determination.

\subsection{Measures of functional connectivity}

In this paper, we compared the traditional measure of MSC to the more recently introduced variant IC. Unlike MSC, IC ignores spectral similarities among 2 signals that occur with zero time delay (Nolte et al., 2004). This has the advantage that it is robust to volume conduction and crosstalk among voxels arising from spatial leakage of the inverse solutions, since these artificial similarities occur with zero time-delay and are therefore removed. IC can therefore not only be used for the localization of corticoperipheral connectivity as done in this study, but also for the localization of cortico-cortical interactions (Guggisberg et al., 2008a). On the other hand, it ignores a portion of true interactions and could therefore lead to less reliable localizations of interactions than MSC. Yet, corticoperipheral coherence is presumed to never occur at zero lag because of the time needed for neural transmission. Indeed, we observed a similar localization accuracy for IC as for MSC in our analyses of cortico-peripheral connectivity.

Many other methods for calculating cortical functional connectivity are available instead of coherence applied here (see e.g. Lachaux et al., 1999; Le Van Quyen et al., 2001; Stam et al., 2003, 2007). While some of them have been compared head-to-head for analyses on the sensor level (Dauwels et al., 2010; Guevara et al., 2005; Le Van Quyen et al., 2001), future studies will need to address performance differences for source connectivity analyses.

\section{Conclusions}

We show that coherent functional sources can be accurately localized and studied with high-resolution EEG systems. The best performance is achieved with precise models of the individual head shape and with inverse solutions that are not susceptible to interference from spatially distant coherent sources.

\section{Acknowledgments}

This study was supported by grant number 320030_129679 of the Swiss National Science Foun- dation. SSD was supported by European Commission grant number PIIF-GA-2008-221097. Cartool is programmed by Denis Brunet and supported by the Center for Biomedical Imaging of Lausanne and Geneva (CIBM). We would like to thank the anonymous reviewers for helpful comments.

Aertsen, A. M., Gerstein, G. L., Habib, M. K., Palm, G., 1989. Dynamics of neuronal firing correlation: modulation of "effective connectivity". J Neurophysiol 61 (5), 900-17.

Ary, J. P., Klein, S. A., Fender, D. H., 1981. Location of sources of evoked scalp potentials: corrections for skull and scalp thicknesses. IEEE Trans Biomed Eng 28 (6), 447-52.

Astolfi, L., Cincotti, F., Mattia, D., Babiloni, C., Carducci, F., Basilisco, A., Rossini, P. M., Salinari, S., Ding, L., Ni, Y., He, B., Babiloni, F., 2005. Assessing cortical functional connectivity by linear inverse estimation and directed transfer function: simulations and application to real data. Clin Neurophysiol 116 (4), 920-32.

Baker, S. N., 2007. Oscillatory interactions between sensorimotor cortex and the periphery. Curr Opin Neurobiol 17, 649-55.

Berg, P., Scherg, M., 1994. A fast method for forward computation of multiple-shell spherical head models. Electroencephalogr Clin Neurophysiol 90 (1), 58-64.

Bianciardi, M., Bianchi, L., Garreffa, G., Abbafati, M., Di Russo, F., Marciani, M. G., Macaluso, E., 2009. Single-epoch analysis of interleaved evoked potentials and fMRI responses during steadystate visual stimulation. Clin Neurophysiol 120 (4), 738-47.

Brookes, M. J., Vrba, J., Robinson, S. E., Stevenson, C. M., Peters, A. M., Barnes, G. R., Hillebrand, A., Morris, P. G., 2008. Optimising experimental design for meg beamformer imaging. Neuroimage 39 (4), 1788-802.

Brunet, D., Murray, M. M., Michel, C. M., 2011. Spatiotemporal analysis of multichannel EEG: CARTOOL. Comput Intell Neurosci 2011, 813870 .

Carter, A. R., Astafiev, S. V., Lang, C. E., Connor, L. T., Rengachary, J., Strube, M. J., Pope, D. L. W., Shulman, G. L., Corbetta, M., 2010. Resting interhemispheric functional magnetic resonance imaging connectivity predicts performance after stroke. Ann Neurol 67 (3), 365-75.

Castellanos, N. P., Paúl, N., Ordóñez, V. E., Demuynck, O., Bajo, R., Campo, P., Bilbao, A., Ortiz, T., del Pozo, F., Maestú, F., 2010. Reorganization of functional connectivity as a correlate of cognitive recovery in acquired brain injury. Brain 133 (Pt 8), 236581.

Dalal, S. S., Guggisberg, A. G., Edwards, E., Sekihara, K., Findlay, A. M., Canolty, R. T., Berger, M. S., Knight, R. T., Barbaro, N. M., Kirsch, H. E., Nagarajan, S. S., 2008. Five-dimensional neuroimaging: Localization of the time-frequency dynamics of cortical activity. Neuroimage 40 (4), 1686-1700.

Dalal, S. S., Zumer, J. M., Guggisberg, A. G., Trumpis, M., Wong, D. D., Sekihara, K., Nagarajan, S. S., 2011. Meg/eeg source reconstruction, statistical evaluation, and visualization with nutmeg. Comput Intell Neurosci 2011, 758973.

Dale, A. M., Liu, A. K., Fischl, B. R., Buckner, R. L., Belliveau, J. W., Lewine, J. D., Halgren, E., 2000. Dynamic statistical parametric mapping: Combining fMRI and MEG for high-resolution imaging of cortical activity for high-resolution imaging of cortical activity. Neuron 26 (1), 55-67.

Dauwels, J., Vialatte, F., Musha, T., Cichocki, A., 2010. A comparative study of synchrony measures for the early diagnosis of Alzheimer's disease based on EEG. NeuroImage 49 (1), 668-93.

de Pasquale, F., Della Penna, S., Snyder, A. Z., Lewis, C., Mantini, D., Marzetti, L., Belardinelli, P., Ciancetta, L., Pizzella, V., Romani, G. L., Corbetta, M., 2010. Temporal dynamics of spontaneous MEG activity in brain networks activity in brain networks. Proc Natl Acad Sci U S A 107 (13), 6040-5.

De Vico Fallani, F., Astolfi, L., Cincotti, F., Mattia, D., Marciani, M. G., Salinari, S., Kurths, J., Gao, S., Cichocki, A., Colosimo, A., Babiloni, F., 2007. Cortical functional connectivity networks 
in normal and spinal cord injured patients: Evaluation by graph analysis. Hum Brain Mapp 28 (12), 1334-46.

Emir, U. E., Bayraktaroglu, Z., Ozturk, C., Ademoglu, A., Demiralp, T., 2008. Changes in bold transients with visual stimuli across 1 $44 \mathrm{~Hz}$. Neurosci Lett 436 (2), 185-8.

Engel, A. K., König, P., Kreiter, A. K., Schillen, T. B., Singer, W. 1992. Temporal coding in the visual cortex: new vistas on integration in the nervous system. Trends Neurosci 15 (6), 218-26.

Fein, G., Raz, J., Brown, F. F., Merrin, E. L., 1988. Common reference coherence data are confounded by power and phase effects. Electroencephalogr Clin Neurophysiol 69 (6), 581-4.

Fender, D. H., 1987. Source localization of brain electrical activity. In: Gevins, A. S., Rémond, A. (Eds.), Handbook of Electroencephalography and Clinical Neurophysiology. Vol 1: Methods of Analysis of Brain Electrical and Magnetic Signals. Elsevier, Amsterdam, pp. 355-403.

Fox, M. D., Snyder, A. Z., Vincent, J. L., Corbetta, M., Van Essen, D. C., Raichle, M. E., 2005. The human brain is intrinsically organized into dynamic, anticorrelated functional networks. Proc Natl Acad Sci U S A 102 (27), 9673-8.

Fransson, P., 2005. Spontaneous low-frequency BOLD signal fluctuations: an fMRI investigation of the resting-state default mode of brain function hypothesis. Hum Brain Mapp 26 (1), 15-29.

Geselowitz, D. B., 1998. The zero of potential. IEEE Eng Med Biol Mag 17 (1), 128-32.

Gray, C. M., König, P., Engel, A. K., Singer, W., 1989. Oscillatory responses in cat visual cortex exhibit inter-columnar synchronization which reflects global stimulus properties. Nature 338 (6213), $334-7$.

Gray, C. M., Singer, W., 1989. Stimulus-specific neuronal oscillations in orientation columns of cat visual cortex. Proc Natl Acad Sci U S A 86 (5), 1698-702.

Gross, J., Kujala, J., Hamalainen, M., Timmermann, L., Schnitzler, A., Salmelin, R., 2001. Dynamic imaging of coherent sources: Studying neural interactions in the human brain. Proc Natl Acad Sci U S A 98 (2), 694-9.

Guevara, R., Velazquez, J. L. P., Nenadovic, V., Wennberg, R., Senjanovic, G., Dominguez, L. G., 2005. Phase synchronization measurements using electroencephalographic recordings: What can we really say about neuronal synchrony? Neuroinformatics 3 (4), 301-14

Guggisberg, A. G., Honma, S. M., Findlay, A. M., Dalal, S. S. Kirsch, H. E., Berger, M. S., Nagarajan, S. S., 2008a. Mapping functional connectivity in patients with brain lesions. Ann Neurol 63 (2), 193-203.

Guggisberg, A. G., Kirsch, H. E., Mantle, M. M., Barbaro, N. M., Nagarajan, S. S., 2008b. Fast oscillations associated with interictal spikes localize the epileptogenic zone in patients with partial epilepsy. Neuroimage. 39 (2), 661-668.

Hadjipapas, A., Hillebrand, A., Holliday, I. E., Singh, K. D., Barnes, G. R., 2005. Assessing interactions of linear and nonlinear neuronal sources using MEG beamformers: a proof of concept. Clin Neurophysiol 116 (6), 1300-13.

Hämäläinen, M. S., Ilmoniemi, R. J., 1994. Interpreting magnetic fields of the brain: minimum norm estimates. Med Biol Eng Comput $32(1), 35-42$.

He, B. J., Snyder, A. Z., Vincent, J. L., Epstein, A., Shulman, G. L., Corbetta, M., 2007. Breakdown of functional connectivity in frontoparietal networks underlies behavioral deficits in spatial neglect. Neuron 53 (6), 905-18.

Hillebrand, A., Barnes, G. R., 2003. The use of anatomical constraints with MEG beamformers. NeuroImage 20 (4), 2302-13.

Jerbi, K., Lachaux, J.-P., N'Diaye, K., Pantazis, D., Leahy, R. M., Garnero, L., Baillet, S., 2007. Coherent neural representation of hand speed in humans revealed by MEG imaging. Proc Natl Acad Sci U S A 104 (18), 7676-81.

Lachaux, J. P., Rodriguez, E., Martinerie, J., Varela, F. J., 1999 Measuring phase synchrony in brain signals. Hum Brain Mapp 8 (4), 194-208.

Le Van Quyen, M., Foucher, J., Lachaux, J., Rodriguez, E., Lutz, A., Martinerie, J., Varela, F. J., 2001. Comparison of Hilbert trans- form and wavelet methods for the analysis of neuronal synchrony. J Neurosci Methods 111 (2), 83-98.

Lin, F. H., Witzel, T., Hamalainen, M. S., Dale, A. M., Belliveau, J. W., Stufflebeam, S. M., 2004. Spectral spatiotemporal imaging of cortical oscillations and interactions in the human brain. Neuroimage 23 (2), 582-95.

Lopes da Silva, F. H., van Lierop, T. H., Schrijer, C. F., van Leeuwen, W. S., 1973. Organization of thalamic and cortical alpha rhythms: Spectra and coherences. Electroencephalogr Clin Neurophysiol 35 (6), 627-39.

Martino, J., Honma, S. M., Findlay, A. M., Guggisberg, A. G., Kirsch, H. E., Berger, M. S., Nagarajan, S. S., 2011. Resting functional connectivity in patients with brain tumors in eloquent areas. Ann Neurol 69, 521-32.

Mima, T., Hallett, M., 1999. Corticomuscular coherence: A review. J Clin Neurophysiol 16 (6), 501-11.

Mitra, P. P., Pesaran, B., 1999. Analysis of dynamic brain imaging data. Biophys J 76 (2), 691-708.

Mosher, J. C., Leahy, R. M., Lewis, P. S., 1999. EEG and MEG: Forward solutions for inverse methods. IEEE Trans Biomed Eng 46 (3), 245-59.

Murthy, V. N., Fetz, E. E., 1992. Coherent 25- to 35-Hz oscillations in the sensorimotor cortex of awake behaving monkeys. Proc Natl Acad Sci U S A 89 (12), 5670-4.

Nolte, G., Bai, O., Wheaton, L., Mari, Z., Vorbach, S., Hallett, M., 2004. Identifying true brain interaction from EEG data using the imaginary part of coherency. Clin Neurophysiol 115 (10), 2292307.

Nunez, P. L., Srinivasan, R., Westdorp, A. F., Wijesinghe, R. S., Tucker, D. M., Silberstein, R. B., Cadusch, P. J., 1997. EEG coherency. I: Statistics, reference electrode, volume conduction, laplacians, cortical imaging, and interpretation at multiple scales. Electroencephalogr Clin Neurophysiol 103 (5), 499-515.

Pascual-Marqui, R. D., 2002. Standardized low-resolution brain electromagnetic tomography (sLORETA): technical details. Methods Find Exp Clin Pharmacol 24 Suppl D, 5-12.

Pascual-Marqui, R. D., Sekihara, K., Brandeis, D., Michel, C., 2009. Imaging the electric neuronal generators of eeg $/ \mathrm{meg}$. In: Michel, C., Koenig, T., Brandeis, D., Gianotti, L., Wackermann, J. (Eds.), Electrical Neuroimaging. Cambridge University Press, Cambridge, pp. $49-77$

Raichle, M. E., Snyder, A. Z., 2007. A default mode of brain function: A brief history of an evolving idea. NeuroImage 37 (4), 1083-90; discussion 1097-9.

Rush, S., Driscoll, D. A., 1968. Current distribution in the brain from surface electrodes. Anesth Analg 47 (6), 717-23.

Schoffelen, J.-M., Gross, J., 2009. Source connectivity analysis with MEG and EEG. Hum Brain Mapp 30 (6), 1857-65.

Schoffelen, J.-M., Oostenveld, R., Fries, P., 2008. Imaging the human motor system's beta-band synchronization during isometric contraction. NeuroImage 41 (2), 437-47.

Sekihara, K., Nagarajan, S. S., Poeppel, D., Marantz, A., 2002. Performance of an MEG adaptive-beamformer technique in the presence of correlated neural activities: effects on signal intensity and time-course estimates. IEEE Trans Biomed Eng 49 (12 Pt 2), $1534-46$.

Sekihara, K., Nagarajan, S. S., Poeppel, D., Marantz, A., 2004. Asymptotic SNR of scalar and vector minimum-variance beamformers for neuromagnetic source reconstruction. IEEE Trans Biomed Eng 51 (10), 1726-34.

Sekihara, K., Nagarajan, S. S., Poeppel, D., Marantz, A., Miyashita, Y., 2001. Reconstructing spatio-temporal activities of neural sources using an MEG vector beamformer technique. IEEE Trans Biomed Eng 48 (7), 760-71.

Sekihara, K., Sahani, M., Nagarajan, S. S., 2005. Localization bias and spatial resolution of adaptive and non-adaptive spatial filters for MEG source reconstruction. NeuroImage 25 (4), 1056-67.

Spinelli, L., Andino, S. G., Lantz, G., Seeck, M., Michel, C. M., 2000. Electromagnetic inverse solutions in anatomically constrained spherical head models. Brain Topogr 13 (2), 115-25.

Srinivasan, R., Winter, W. R., Ding, J., Nunez, P. L., 2007. EEG and 
MEG coherence: Measures of functional connectivity at distinct spatial scales of neocortical dynamics. J Neurosci Methods 166 (1), $41-52$.

Stam, C. J., Breakspear, M., van Cappellen van Walsum, A.-M., van Dijk, B. W., 2003. Nonlinear synchronization in EEG and whole-head MEG recordings of healthy subjects. Hum Brain Mapp $19(2), 63-78$

Stam, C. J., Nolte, G., Daffertshofer, A., 2007. Phase lag index Assessment of functional connectivity from multi channel EEG and MEG with diminished bias from common sources. Hum Brain Mapp 28 (11), 1178-93.

Steinsträter, O., Sillekens, S., Junghoefer, M., Burger, M., Wolters, C. H., 2010. Sensitivity of beamformer source analysis to deficiencies in forward modeling. Hum Brain Mapp 31 (12), 1907-27.

Stenroos, M., Mäntynen, V., Nenonen, J., 2007. A Matlab library for solving quasi-static volume conduction problems using the boundary element method. Comput Methods Programs Biomed 88 (3), 256-63.

Thatcher, R. W., Krause, P. J., Hrybyk, M., 1986. Cortico-cortical associations and EEG coherence: a two-compartmental model. Electroencephalogr Clin Neurophysiol 64 (2), 123-43.

Van Veen, B. D., van Drongelen, W., Yuchtman, M., Suzuki, A. 1997. Localization of brain electrical activity via linearly constrained minimum variance spatial filtering. IEEE Trans Biomed Eng $44(9), 867-80$.

Varela, F., Lachaux, J. P., Rodriguez, E., Martinerie, J., 2001. The brainweb: phase synchronization and large-scale integration. Nat Rev Neurosci 2 (4), 229-39.

Victor, J. D., Purpura, K., Katz, E., Mao, B., 1994. Population encoding of spatial frequency, orientation, and color in macaque V1. J Neurophysiol 72 (5), 2151-66.

Vincent, J. L., Patel, G. H., Fox, M. D., Snyder, A. Z., Baker, J. T., Van Essen, D. C., Zempel, J. M., Snyder, L. H., Corbetta, M. Raichle, M. E., May 2007. Intrinsic functional architecture in the anaesthetized monkey brain. Nature 447 (7140), 83-6.

Vrba, J., Robinson, S., 2001. Signal processing in magnetoencephalography. Methods 25 (2), 249-271.

Witham, C. L., Wang, M., Baker, S. N., 2010. Corticomuscular coherence between motor cortex, somatosensory areas and forearm muscles in the monkey. Front Syst Neurosci 4, 38 .

Wong, D. D. E., Gordon, K. A., Dec 2009. Beamformer suppression of cochlear implant artifacts in an electroencephalography dataset. IEEE Trans Biomed Eng 56 (12), 2851-7. 